2011-01

\title{
Solutions and Open Challenges for the Symbol Grounding Problem
}

Cangelosi, A

http://hdl.handle.net/10026.1/3595

International Journal of Signs and Semiotic Systems (IJSSS)

All content in PEARL is protected by copyright law. Author manuscripts are made available in accordance with publisher policies. Please cite only the published version using the details provided on the item record or document. In the absence of an open licence (e.g. Creative Commons), permissions for further reuse of content should be sought from the publisher or author. 


\title{
Solutions and Open Challenges for the Symbol Grounding Problem
}

\section{Angelo Cangelosi}

\author{
Adaptive Behaviour \& Cognition Lab \\ Centre for Robotics and Neural Systems \\ University of Plymouth \\ Drake Circus, Plymouth PL4 8AA (UK) \\ Tel. +44 1752 586217, Fax +44 1752586300 \\ acangelosi@plymouth.ac.uk \\ http://www.tech.plym.ac.uk/soc/staff/angelo
}

\begin{abstract}
This article discusses the current progress and solutions to the symbol grounding problem, indentify specifically which aspects of the problem have been already addressed, and which other issues and scientific challenges still require investigation. In particular, the paper suggests that of the various aspects of the symbol grounding problems, the one that still require significant research is that on the transition from indexical representations to symbol-symbol relationships. This analysis initiated a debate and solicited commentaries from experts in the field, to gather consensus on actual progress and achievements and to identify the challenges still open in the symbol grounding problem.
\end{abstract}

\section{Keywords}

Symbol grounding, embodiment, cognitive robotics, developmental robotics

\section{Biographical sketch}

Angelo Cangelosi is Professor of Artificial Intelligence and Cognition at the University of Plymouth (UK), where he leads the Centre for Robotics and Neural Systems. He obtained a PhD in psychology and computational modeling in 1997 from the University of Genoa, whilst also working as visiting scholar at the National Research Council (Rome), the University of California San Diego (USA) and the University of Southampton (UK). He has produced more than 140 scientific publications, and has been awarded numerous research grants from UK and international funding agencies. He is coordinator of the EU FP7 project "ITALK" (italkproject.org) and the "RobotDoC" Marie Curie doctoral network in developmental robotics (robotdoc.org). 


\section{MAIN TEXT}

The grounding of symbols, i.e. the process by which symbols (such as words) are linked to the agent's own semantic representation of the world, is an issue of crucial importance to the field of cognitive science, raging from philosophy and semiotics, to artificial intelligence, and to cognitive robotics. For example, in cognitive and developmental robotics, in the last decades there has been a tremendous increase in new models of the evolution of communication and the developmental acquisition of language. These models directly regard the issue of symbol grounding, since robotic agents are trained to use symbols that often refer to entities and states in the robot's own world.

In the literature on artificial intelligence, cognitive science and philosophy, there has been extensive discussion on the symbol grounding problem. Different views on the importance of the symbol grounding have been proposed. On one extreme, we find the so called "symbolic" approaches that practically ignore the cognitive significance of such an issue (e.g. Fodor 1983, Chomsky 1965). This is the case of classical GOFAI (Good Old Fashion Artificial Intelligence) approaches, based on symbolic methods such as logic, where the meaning or a linguistic symbol (word) is simply represented by another symbol (meaning), without having to deal of how meanings have formed and have been associated to words. On the other hand, new "embodied" approaches to cognition acknowledge the importance of grounding symbols through the agent's interaction with the environment. However, even in this field, there have been suggestions that the problem has practically been solved (Steels 2008), thus appearing to suggest that the symbol grounding problem is not important anymore.

In this target article, I am going to suggest a different analysis of the current progress on symbol grounding, indentify specifically which aspects of the problem have been already addressed, and which other issues still require investigation. This analysis will initiate a debate and solicit commentaries from experts in the field, to gather consensus on actual progress, achievements and open challenges in the symbol grounding problem.

To assess better the current state of the art on the Symbol Ground Problem, I will use the definition and discussion of the problem originally given by Stevan Harnad in his seminal 1990 article "The Symbol Grounding Problem". Here Harnad explains that the symbol grounding problem refers to the capability of natural and artificial cognitive agents to acquire an intrinsic link (autonomous, we would say in nowadays robotics terminology) between internal symbolic representations and some referents in the external word or internal states. In addition, Harnad explicitly proposes a definition of a symbol that requires the existence of logical links (e.g. syntactic) between the symbols themselves. It is thanks to these inter-symbol links, its associated symbol manipulation processes, and the symbol grounding transfer mechanism (Cangelosi \& Riga 2006) that a symbolic system like human language can exist. The symbol-symbol link is the main property that differentiates a real 
symbol from an index, as in Peirce's semiotics. These symbolic (e.g. syntactic) links also support the phenomena of productivity and generativity in language, and contribute to the grounding of abstract concepts and symbols (Barsalou 1999). Finally, an important component of the symbol grounding problem is the social and cultural dimension, that is the role of social interaction in the sharing of symbols (a.k.a. the external/social symbol grounding problem, as in Cangelosi 2006; Vogt 1997; Vogt \& Divina, 2007).

To summarise, we can say that there are three sub-problems in the development of a grounded symbol system:

(i) how can a cognitive agent autonomously link symbols to referents in the world such as objects, events and internal and external states;

(ii) how can an agent autonomously create a set of symbol-symbol relationships and the associated transition from an indexical system to a proper symbol system;

(iii) how can a society of agents autonomously develop a shared set of symbols.

When we look at the current cognitive agent models of language learning (e.g. Cangelosi \& Parisi, 2002; Wagner et al. 2003; Lyon et al. 2007), I believe that much progress has been achieved in the first and third sub-problems (individual and social grounding of symbols), whilst I believe that for the second sub-problem (autonomous creating of symbol-symbol relationships) much still needs to be done. I therefore agree with Steels (2008) that much has been done on the robotic and cognitive modelling of the symbol grounding problem, but only when we consider the two subproblems (i) and (iii), "we now understand enough to create experiments in which groups of agents self-organize symbolic systems that are grounded in their interactions with the world and others" (Steels 2008: page 240).

For example, when we consider the well known "Talking Heads" model of the emergence of communication in robotic agents (Steels, 1999; Kaplan \& Steels, 2002), we have a demonstration that each individual robot can autonomously create categorical representations of the world (a white board with a variable combination of coloured shapes). Using the technique of discrimination trees, each agent creates categories defined by specific patters of boundaries of color, shape and spatial values. In addition, these perceptual categories become associated with words that the agents use to communicate with each other in "language games" (Steels 2001). That is, these categories constitute the internal meanings of the agent's communication lexicon. What is crucial in this model is that the association of a specific perceptual category with a word is mediated by the social interaction with the other agents. This therefore leads to the cultural evolution of a shared set of words (symbols). Such a modelling approach has been extended to human-robot communication systems, such as for the training of the AIBO pet dog to name objects (Kaplan \& Steels, 2000).

In a multi-agent model of the evolutionary emergence of communication, similar mechanisms for the grounding of shared lexicons have been utilised (Cangelosi 2001). Simulated cognitive agents live in a 2D world to perform foraging tasks and learn to differentiate between edible mushrooms (that when eaten increase the chances of survival and reproduction) and toadstools (which cause a decrease of fitness). Agents autonomously learn to categorize foods in edible and inedible 
categories through their own neural network that controls the agent's perceptual and motor system. In addition, as agents are allowed to communicate with each-other, this cause the gradual evolution of a shared lexicon. The activation patterns in the agents' neural network (hidden units) constitute the distributed semantic representations of the food categories, and at the same time they support the linguistic comprehension and production capability of the agents. In fact, analyses of the agent's internal representations suggest that the evolutionary pressure to evolve a shared lexicon is explained by the categorical perception effects of these representations.

The above examples are only some of the numerous cognitive agents and robotics models of the evolution and acquisition of language that have demonstrated the mechanisms for the autonomous grounding of symbols and the emergence of shared lexicons (e.g. Loula et al. 2010; Marocco et al. in press; Roy 2005; Vogt 2005; Vogt \& Divina 2007). At the same time, as Steels also acknowledges, it is also true that we do not yet have a full understanding of all the mechanisms in grounding, such as on the nature, role and making of internal symbolic representations.

As for the sub-problem (ii), i.e. the transition from a communication systems based on indices (e.g. labels, animal communication, early child language learning) to that of a full symbolic system (e.g. adult human languages), I believe that the problem has not really been solved at all, and much needs to be done.

Most computational models of syntactic learning and evolution, based on the grounding principles as defined above, have only loosely addressed the issue of the autonomous development of symbol-symbol relationships. In very few cases, grounded models of the evolution of communication use limited syntactic constructs that partially resemble grammatical constructs (e.g. Cangelosi 2001; Cangelosi et al. 2000). In other cases, robotics models of the evolution of language use pre-defined, symbolic approaches to represent symbol-symbol relationships, e.g. by assuming the pre-existence of semantic and syntactic categories in the agent's cognitive system. This is however in contrast with the grounding principles, as the aim of a cognitive agent model of the evolution of language is to discover the evolutionary, neural and cognitive mechanisms that lead to the emergence of syntactic symbolsymbol representations.

I invite my colleagues to comment on the state of the art on the symbol grounding problem in cognitive science and robotic models of communication and language, and on their view on the importance (or not!) of the symbol grounding problem. I suggest below some open challenges for future research that I believe are crucial for our understanding of the symbol grounding phenomena, and I welcome suggestions for other important, unsolved challenges in this field.

(1) Is the symbol grounding problem, and the three sub-problems as identified above, still a real crucial issue in cognitive robotics research? And if the problem appears to have been solved, as some have suggested, why is it that so far we have failed at building robots that can learn language like children do?

(2) What are the processes that lead to the transition from indexical communication system to a full symbolic system such as language? Is there a continuum between indices (labels) and symbols (words), or is the transition 
qualitative and sudden? What known phenomena in language origins theories, and in developmental studies, should be included in developmental and evolutionary robotics model of language?

(3) Notwithstanding the importance of the grounding problem, there are still various approaches in the agent/robot language learning/evolution literature that practically ignore the process of grounding and use a symbolic-only approach to the definition of meanings and words. Do these symbolic approaches really give an important contribution to our understading of human cognition, of should all models of language learning be based solely on grounding mechanisms?

(4) Does cognitive development really plays an important role in symbol grounding and acquisition, or is it just an epiphenomenon of no crucial importance to the understanding of human cognition? Some key findings and experiments show that infants have strong specific biases that allow them to learn very easily language. And most attempts at building robots without these biases have failed so far to learn realistically complex concepts/semantic categories. Is the symbol grounding problem just a matter of using and identifying such biases in robotics language models?

(5) What kind of robotic experiment would constitute a real breakthrough to advance the debate on symbol grounding, and what kind of principle and ideas are still unexplored?

(6) What are the properties and differences of internal representations beyond both indexical and symbolic systems? Or are representation issues not really crucial, as a pure sensorimotor modelling approach would not require any internal representation capability?

(7) How can we model the grounding of abstract concepts such as beauty, happiness, time. Or is the grounding approach inconsistent with the study of higher-order symbolic capabilities?

(8) What are the grounding components in the acquisition and use of function words (such as verb preposition "to", as in verbs "to go", "if", "the"), of number concepts/words, and of morphology and other syntactic properties.

(9) How can we model the grounding phenomena studies through empirical investigations of language embodiment (Barsalou 1999; Glenberg \& Kaschak 2002; Pecher \& Zwaan 2005)?

\section{Acknowledgements}

The author wishes to acknowledge the support of the EU FP7 projects ITALK and ROBOTDOC.

A shorter version of this target article, and some of the commentaries here included (Harnad, Steels, Madden et al. Sloman, Muller, Cowley), have appeared in the AMD Newsletter (vol. 7, April 2010) of the IEEE-CIS Autonomous Mental Development Technical Committee http://www.cse.msu.edu/amdtc/amdnl/

\section{References}

Barsalou, L. 1999, "Perceptual symbol systems". Behavioral and Brain Sciences, 22: 577-609.

Cangelosi A. (2006). The grounding and sharing of symbols. Pragmatics and Cognition, 14(2), 275285

Cangelosi A., Greco A. \& Harnad S. (2000). From robotic toil to symbolic theft: Grounding transfer from entry-level to higher-level categories. Connection Science, 12(2), 143-162 
Cangelosi A, Riga T (2006). An embodied model for sensorimotor grounding and grounding transfer: Experiments with epigenetic robots, Cognitive Science, 30(4), 673-689

Cangelosi A. \& Parisi D. (Eds.) (2002). Simulating the Evolution of Language. London: Springer.

Cangelosi A. (2001). Evolution of communication and language using signals, symbols and words. IEEE Transactions on Evolutionary Computation. 5(2), 93-101

Chomsky N. (1965). Aspects of the Theory of Syntax. Cambridge: The MIT Press.

Fodor, J.A. (1983). The modularity of mind: An essay on faculty psychology. Cambridge, MA: Bradford Books, MIT Press.

Glenberg A. \& Kaschak M. (2002). Grounding language in action. Phychonomic Bulletin \& Review, 9(3):558-565

Harnad, S. (1990) The Symbol Grounding Problem. Physica D 42: 335-346.

Loula, A., Gudwin, R., El-Hani, C., and Queiroz, J. (2010) Emergence of self-organized symbol-based communication in artificial creatures. Cognitive Systems Research, 11(2):131--147.

Lyon C., Nehaniv C.L., Cangelosi A. (Eds.) (2007). Emergence of Communication and Language. London: Springer.

Marocco D., Cangelosi A., Fischer K., Belpaeme T. (in press). Grounding action words in the sensorimotor interaction with the world: Experiments with a simulated iCub humanoid robot. Frontiers in Neurorobotics

Pecher D. \& Zwaan R.A. (2005). Grounding Cognition: The Role of Perception and Action in Memory, Language, and Thinking: Cambridge University Press.

Roy, D. (2005). Grounding words in perception and action: Insights from computational models. Trends in Cognitive Science, 9 (8): 389-96

Steels, L. (1999). The Talking Heads Experiment Volume 1. Words and Meaning; Laboratorium, Antwerpen.

Steels L. (2001). Language games for autonomous robots. IEEE Intelligent Systems, 16)5), 16-22.

Steels, L. (2008) The symbol grounding problem has been solved. So what's next? In de Vega, M., (eds.), Symbols and Embodiment: Debates on Meaning and Cognition. Oxford: Oxford University Press. pp. 223-244.

Steels, L. and Kaplan, F., (2000). AIBO's first words, the social learning of language and meaning, Evolution of Communication, 4 (1): 3-32

Steels, L. and Kaplan, F. (2002) Bootstrapping grounded word semantics. In Briscoe,T. Linguistic evolution through language acquisition: formal and computational models, p. 53-73, Cambdrige University Press, Cambridge, UK.

Vogt P. (2002). Physical symbol grounding. Cognitive Systems Research, 3(3):429--457.

Vogt, P. (2005) The emergence of compositional structures in perceptually grounded language games. Artificial Intelligence, 167(1-2):206--242.

Vogt, P. and Divina, F. (2007) Social symbol grounding and language evolution. Interaction Studies, $8(1): 31--52$.

Wagner, K., Reggia, J. A., Uriagereka, J., \& Wilkinson, G. S. (2003) Progress in the simulation of emergent communication and language. Adaptive Behavior, 11(1):37--69. 